with diverse patients, and support the theoretical premise of the intervention.

\section{THE EFFECTS OF RTMS ON PSYCHOMOTOR RETARDATION IN ELDERLY DEPRESSION}

V. Thomas-Ollivier ${ }^{1}$, E. Foyer ${ }^{1,2}$, S. Bulteau ${ }^{2}$, A. Sauvaget ${ }^{2}$, T. Deschamps ${ }^{1}$ 1. Laboratory "Movement, Interactions, Performance" (E.A. 4334), University of Nantes, France, Nantes, France, 2. Department of "Addictology \& Consultation-Liaison psychiatry", Nantes University Hospital, Nantes, France, Nantes, France

Depression-related psychomotor retardation (PMR) is often misinterpreted as the age-related slowing, playing down the importance of depression in aging population. Thus we tested the PMR-related cognitive flexibility by assessing the verbal fluency performance in healthy older controls $(n=18$; mean age: $61.7 \pm 9.23$ years) and in age-matched patients with major depressive disorder (MDD) $(n=29$; mean age: $57.1 \pm 12.4$ years). We also examined the antidepressant effects of repetitive transcranial magnetic stimulation (rTMS) in eligible MDD patients ( $n=17)$, evidenced by an expected verbal fluency improvement. Three scores were assessed for semantic and phonemic fluency tests: (1) total number of words generated excluding preservative and intrusive errors; (2) number of switches; and (3) mean cluster size. The results clearly showed that PMR in geriatric depression differed from the age related slowing. Significant differences between groups in cluster size $(p<0.05)$ and percent of preservative errors $(p<0.04)$ were found for the phonemic fluency performance. In addition to significant improvement of the depression level $(\mathrm{p}<0.001)$ and the PMR score $(\mathrm{p}<0.001)$ after the rTMS treatment, the results showed a nonsignificant trend toward an increasing verbal fluency performance. Overall, the present study confirms the negative influence of depression on verbal episodic memory performance, regardless of age. But the depression-related deficits in cognitive flexibility seem not to be associated with the PMR scores before and after the rTMS treatment, challenging the possible validation of verbal fluency performance as one of relevant hallmark of PMR.

\section{THE ASSOCIATION BETWEEN PHYSICAL ACTIVITY AND DEPRESSION IN OLDER ADULTS WITH PARKINSON'S DISEASE}

M. Ventura ${ }^{1}$, J.K. Johnson ${ }^{1}$, D.E. Barnes ${ }^{1,2}, 1$. University of California, San Francisco, San Francisco, California, 2. San Francisco VA Medical Center, San Francisco, California

It is important to understand the associations between physical activity (PA) and mood in the early stages of Parkinson's disease (PD) progression. The purpose of this study was to explore the relationship between PA and depression in newly diagnosed, untreated individuals with PD. We analyzed data from the Parkinson Progression Markers Initiative and examined depression (Geriatric Depression Scale; GDS) in 260 de novo individuals with PD. Participants with a GDS score $\leq 5$ were considered "not depressed" and those with a GDS score $>5$ were considered "depressed." We also examined scores on the Montreal Cognitive Assessment and self-reported levels of PA (Physical Activity Scale for the Elderly). Physical activity including light, moderate and strenuous PA, were tallied to create summary scores for frequency (days/week) and duration (hours/day). Thirty-four percent of study participants reported being depressed at baseline. Those who were depressed had fewer years of education ( 15 vs. 16 years, $\mathrm{p}=0.02$ ) but did not differ significantly by age, sex or cognitive function compared to those with no depression. Study participants engaged in PA for an average of $1.3 \pm 0.4$ days/week for $2.2 \pm 0.4$ hours. There were no significant differences in PA levels between those who were depressed or not depressed. Depressive symptoms were high in this cohort of patients with newly diagnosed, untreated Parkinson's disease. However, we did not observe an association between physical activity and depressive symptoms cross-sectionally. Future studies should examine the longitudinal association between physical activity and depressive symptoms in this cohort.

\section{THE INTERPLAY OF LIFE EVENTS, RELIGIOUS EXPRESSION, AND SOCIAL SUPPORT WITH DEPRESSION}

A.M. Ogletree, R. Blieszner, L.P. Sands, Human

Development, Virginia Tech, Blacksburg, Virginia

Based on Pearlin's stress process model, we examined relationships among religious expression/participation, social support, and depressive symptoms in adults who experienced significant life events (SLEs) within the past year. Data came from the ORANJ BOWL study and included 5,468 randomly-dialed New Jersey residents aged 50-74 in 2008. We explored which events were the strongest indicators of the SLE construct and most associated with higher CES-D scores. We hypothesized more depressive symptoms among individuals with more stressful events, less social support, and lower religious expression. Religiosity was measured using 6 items from the Multidimensional Measurement of Religiousness/Spirituality. The 10-item CES-D indexed depressive symptoms; life events were recorded on a 14-item Cochrane-Robertson Life Event Inventory; perceived social support was measured using 4 items. Structural equation modeling assessed construct factor loadings and hypothesized associations; fit indices revealed good model fit to the data $\left(x^{2}=3637.32, p=0.000, d f=221\right.$; RMSEA=0.053, $\mathrm{NNFI}=0.911, \mathrm{CFI}=0.922, \mathrm{SRMR}=0.047)$. The strongest indicators of SLE were three items reflecting relational family events. Maximum likelihood estimates partially supported the hypotheses: individuals with more stressful events, lower public religious expression, and less perceived social support reported significantly more depressive symptoms. However, individuals with more private religious expression also had more depressive symptoms. These findings reveal that private versus public religious expression may have different roles in coping with significant life events.

\section{RELIGIOSITY AND DEPRESSIVE SYMPTOMS IN OLDER ADULTS COMPARED TO YOUNGER ADULTS: MODERATION BY AGE}

M.A. Stearns, E.D. Lantz, D.K. Nadorff, Mississippi State

University, Starkville, Mississippi

Overall, religious individuals are more likely to have a positive life view, and are less likely to suffer from depressive symptoms. Given the high prevalence of depressive symptoms among older adults, it is important to identify possible protective factors. Thus, the current study examines the 
effect of age on the relation between religiosity and depressive symptoms.

The sample $(N=316)$ was recruited using MTURK. Measures included age, the Center for Epidemiological Studies Depression Scale (CESD), and the StearnsMcKinney Assessment of Religious Traits (SMART). A large variance of ages was sought and ranged from 19 to 67. As expected, we found that the older adults aged 50 and older $(M=230.35, S D=106.97)$ were more religious than younger adults aged 18-30 $(M=230.35, S D=106.97, t$ $(1,437)=-6.07, p<.001)$. Additionally, younger adults $(M=18.40, S D=13.72)$ reported more depressive symptoms than the older adults $(M=9.43, S D=10.45, t(1$, $67.975)=3.16, p=.002)$.

Moderation analyses were conducted using SPSS' Process macro on the entire data set. The interaction between religiosity and age just missed significance in predicting depressive symptoms, $t(1,315)=-1.74, p=.08$. There was not a significant relation of religiosity on depressive symptoms for those one or more standard deviations below the mean age $(t=0.26, p=.80)$, whereas there was a significant relation for those one standard deviation above the mean $(t=-2.40$, $p=.02)$. Implications, future directions, and limitations are discussed.

\section{PERSONALITY, BOREDOM, AND COMPENSATORY USE OF TV IN THE HEALTH AND RETIREMENT STUDY}

P.A. Fox ${ }^{1}$, S.T. Mejia ${ }^{2}$, K.A. Hooker ${ }^{3}$, 1. DePaul University, Chicago, Illinois, 2. University of Michigan, Ann Arbor, Michigan, 3. Oregon State University, Corvallis, Oregon

In addition to being a sedentary activity with health and well-being correlates, TV watching is also one of the most commonly reported daily activities among older adults. Despite especially high variability in time spent watching TV in this age group, personality has not been well integrated into explanations of that variation, or the study of conditions under which TV watching may serve as a compensation strategy when other activities are not available (Van der Goot, 2015; Baltes \& Baltes, 1990). We used 2012 Health and Retirement Study data $(\mathrm{N}=4,655)$ to examine conscientiousness, neuroticism, perceived boredom yesterday, and hours spent watching TV yesterday to examine how personality shapes time spent on this activity and the importance of perception that one does not have other things to do. As expected, lower conscientiousness and higher neuroticism were associated with watching TV longer as well as lower experienced well-being during TV watching (Net positive affect while watching TV; $\mathrm{B}_{\mathrm{C}}=.54$, $\mathrm{B}_{\mathrm{N}}=-.42$, both $\mathrm{p}<.001$; Smith et al., 2014, p. 63). However, high conscientiousness individuals watched less TV only if the day was low in boredom. For neuroticism but not conscientiousness, there was a significant indirect effect via boredom, and unexpectedly, the remaining association of neuroticism to TV time was negative $(\mathrm{B}=-.17, \mathrm{p}<.05)$. This suggests that beyond the boredom-TV pathway, neuroticism may be associated with less TV watching. Results partially supported the connection of conscientiousness to selective TV watching, and neuroticism to compensatory use of TV.

\section{MALADAPTIVE PERSONALITY AND SOCIAL ROLE IMPAIRMENT IN DEPRESSED OLDER ADULTS IN PRIMARY CARE}

A. Romirowsky ${ }^{1}$, R.A. Zweig ${ }^{1,2}$, L. Glick ${ }^{1}$, J. Sirey ${ }^{3}, 1$. Yeshiva University, Bronx, New York, 2. Albert Einstein College of Medicine, Bronx, New York, 3. Weill Cornell Medicine, White Plains, New York

Personality pathology is associated with impaired social functioning in adults, though further evidence is needed to examine the individual contributions of personality traits and processes to social functioning in depressed older adults. This study is a secondary analysis examining the relationship between maladaptive personality traits and processes and social role impairment in depressed older adults in primary care $(\mathrm{N}=56)$. Participants were $77 \%$ female and ranged in age between 55-89 $(M=66.82, S D=8.75)$. Personality pathology was measured both by maladaptive traits (NEO-FFI) and processes (Inventory of Interpersonal Problems; IIP-15). Individual variable as well as combined predictive models of social role impairment were examined. Higher neuroticism $(\beta=0.30, p<.05)$, lower agreeableness $(\beta=-0.35 p<.001)$ and higher IIP-15 $(\beta=0.28, p<.01)$ scores predicted greater impairment in social role functioning. Categorical analyses of these variables found significant differences in social role functioning between individuals with high and low levels of agreeableness $(p=.05)$ and IIP-15 scores $(p<.01)$. All moderation analyses were non-significant, but a combined predictive model of neuroticism and IIP-15 scores predicted unique variance in social role impairment $(R 2=.71)$. These findings highlight the importance of accounting for personality pathology, as measured by both traits and processes, in the assessment and treatment of older adults with depression. In addition, these findings lend support for continued research into both linear and non-linear relationships between personality pathology and domains of functional impairment in older adults.

\section{PERSONALITY AND ALL-CAUSE MORTALITY: COMBINED ANALYSIS BETWEEN NEUROTICISM AND EXTRAVERSION}

H. Iwasa ${ }^{1}$, Y. Masui ${ }^{2}$, Y. Gondo ${ }^{3}$, H. Inagaki², Y. Yoshida ${ }^{2}$, 1. Fukushima Medical University, Fukushima-shi, Japan, 2.

Tokyo Metropolitan Institute of Gerontology, Tokyo, Japan, 3. Osaka University, Osaka, Japan

Purpose: Although recent studies have confirmed a relationship solely between personality traits and mortality, combinations of personality have been not fully investigated. This study explored whether a combination of personality traits was related to all-cause mortality among the middleaged and elderly.

Methods: A total sample of 1050 men and 1424 women aged 52 to 77 years were examined at the baseline. The Eysenck Personality Inventory was administered to assess neuroticism and extraversion. We created three categories approximating the three tertiles of each personality trait score. We also combined the two scores and created four groups: Gloomy $(\mathrm{N}+\mathrm{E}-)$, Passionate $(\mathrm{N}+\mathrm{E}+)$, Low-keyed (N-E-), and Resilient (N-E+).

Results: During the 7-year follow-up period, 187 persons $(7.6 \%, 119$ men and 68 women) died, and 261 people $(10.5 \%$, 112 men and 149 women) moved away from the target region. 